\title{
Effect of acute exercise on the levels of salivary cortisol, tumor necrosis factor- $\alpha$ and nitric oxide
}

\author{
Zamzy A. Rahman ${ }^{1)}$, Nizam Abdullah ${ }^{2)}$, Rabindarjeet Singh ${ }^{1,3)}$ \\ and Wihaskoro Sosroseno ${ }^{4)}$
}

\author{
${ }^{1)}$ Sport Science Unit, Department of Physiology, University of Sains Malaysia, Kota Bharu, Malaysia \\ ${ }^{2)}$ College of Dentistry, University of Sharjah, Sharjah, United Arab Emirates \\ ${ }^{3}$ Institute of Advance Medical and Dental Science, University of Sains Malaysia, Pulau Penang, Malaysia \\ ${ }^{4)}$ Faculty of Dentistry, The Asian Institute of Medicine, Science and Technology (AIMST) University, \\ Kedah, Malaysia
}

(Received 22 June and accepted 3 December 2009)

\begin{abstract}
The aim of the present study was to assess the levels of salivary cortisol, tumor necrosis factoralpha (TNF- $\alpha$ ) and nitric oxide (NO) before, during and after acute exercise. Acute exercise was induced using a standard treadmill test with Bruce protocol in ten physically active male participants. Unstimulated saliva was collected before, during and after exercise. The levels of salivary cortisol and TNF- $\alpha$ were assessed by enzyme immunoassays. Salivary NO was determined by the Griess reagent. The results showed that both salivary cortisol and TNF- $\alpha$ increased and peaked at 14 min during exercise and then decreased. The levels of NO were increased up to $1 \mathrm{~h}$ after exercise and subsequently lowered after $24 \mathrm{~h}$. The results of the present study suggest that acute exercise may induce high levels of salivary cortisol, TNF- $\alpha$ and NO. (J Oral Sci 52, 133-136, 2010)
\end{abstract}

Keywords: cortisol; exercise; nitric oxide; saliva; TNF- $\alpha$.

\section{Introduction}

Acute exercise leads to rapid adaptation of the cardiovascular, endocrine, musculoskeletal and central nervous

Correspondence to Dr. Wihaskoro Sosroseno, Faculty of Dentistry, AIMST University, Semeling, Bedong 08100, Kedah Darul Aman, Malaysia

Tel: +60-4-4298532

Fax: +60-4-4422887

E-mail: wsosroseno@yahoo.com systems and, therefore, to physiological disturbances. Whilst peripheral blood remains to be the most useful biological fluid to assess the pathophysiology of acute exercise in humans, saliva has been increasingly investigated as an easily accessible fluid that may indicate systemic alterations due to exercise (1). Previous reports have indicated that acute exercise increases cortisol levels in saliva $(2,3)$. Therefore, assessment of salivary cortisol levels is nowadays a common practice to detect the effect of exercise on human physiology.

Tumor necrosis factor-alpha (TNF- $\alpha$ ) is one of the pro-inflammatory cytokines that plays a crucial role in the host response to acute exercise. Previous studies demonstrated that the plasma levels of TNF- $\alpha$ are significantly increased after acute exercise in healthy humans $(4,5)$, suggesting that acute exercise may augment the host susceptibility to developing an inflammatory reaction. However, reports showing the levels of this cytokine in saliva before, during and after acute exercise are lacking.

Nitric oxide (NO) is a gaseous molecule that regulates blood flow and both muscle contraction and metabolism during exercise (6). The plasma levels of NO increase after acute exercise in humans $(7,8)$. Unfortunately, studies that determined the alterations in levels of salivary NO due to acute exercise are also lacking. A study carried out in athletes by Panossian and colleagues (9) indicated that salivary NO is increased in early acute exercise. However, this finding remains to be confirmed.

Since acute exercise has been shown to alter the levels of plasma cortisol, TNF- $\alpha$ and NO, the aim of the present study was to test a hypothesis that the levels of these 
parameters in saliva may also be affected by acute exercise in physically active subjects.

\section{Materials and Methods}

Ten physically active, non-smoking male subjects were enrolled in the present study after providing written informed consent. All subjects received professional tooth cleaning and instruction on proper oral hygiene to ensure good oral hygiene prior to the experiment. A standard treadmill test with Bruce protocol was performed as a method to induce acute exercise (10). Heart rate and subjective evaluation of exercise intensity using Perceived Exertion (RPE) scale (11) were continuously monitored before and during exercise. Unstimulated whole saliva was collected by expectoration into a sterile tube and centrifuged at 1,000 rpm for $2 \mathrm{~min}$. The supernatant was collected and stored at $-4^{\circ} \mathrm{C}$ until use. The levels of cortisol (DSL, Webster, TX, USA) and TNF- $\alpha$ (Bender Medsystems, Vienna, Austria) in undiluted saliva were assessed by commercially available enzyme immunoassay kits, according to the manufacturers' instructions. The

Table 1 Physical and physiological characteristics of the subjects

\begin{tabular}{ll}
\hline Parameter & Mean $(\mathrm{SD})$ \\
\hline Subjects $(n=10)$ & $21.1(2.34)$ \\
Age $(\mathrm{yr})$ & $63.0(6.63)$ \\
Weight $(\mathrm{kg})$ before exercise & $62.5(6.45)$ \\
Weight $(\mathrm{kg})$ after exercise & $169.6(3.93)$ \\
Height $(\mathrm{cm})$ & $21.3(2.10)$ \\
Body mass index $(\mathrm{BMI})$ & $6396.7(470.1)$ \\
Basal metabolic rate $(\mathrm{Kcal})$ & $121.9(6.33)$ \\
Systolic pressure $(\mathrm{mmHg})$ & $74.2(6.35)$ \\
Diastolic pressure $\left(\mathrm{mmHg}^{-1}\right)$ & $43.58(3.32)$ \\
$\mathrm{VO}_{2} \max \left(\mathrm{ml} \cdot \mathrm{kg}^{-1} \cdot \mathrm{min}^{-1}\right)$ & \\
\hline
\end{tabular}

$* \mathrm{VO}_{2} \mathrm{max}=$ maximum oxygen uptake levels of nitrite, a byproduct of NO, were determined by the Griess reagent. Briefly, $100 \mu$ l of undiluted saliva was mixed with equal volume of the Griess reagent $(1 \%$ sulfanilamide, $0.1 \%$ naphthlethylenediamine dihydrochloride in $2.5 \%$ phosphoric acid) and the color was read in an automated plate reader (BioTek Instruments Inc., Winooski, VT, USA) at $540 \mathrm{~nm}$. Sodium nitrite was used to prepare a standard curve for nitrite levels. All reagents for NO assays were purchased from Sigma (St. Louis, MO, USA). The present study was approved by the Research and Ethics committee, School of Medical Science, University Sains Malaysia. Data were statistically analyzed by a repeat measurement test using a statistical analysis software package (SPSS, Chicago, IL, USA).

\section{Results}

Physical and physiological characteristics of the subjects are shown in Table 1. Since none of the subjects could complete the treadmill test, exercise was terminated at 15 min. Heart rate and the RPE scale at 15 min of exercise were $185.9 \pm 37.14$ beats/min and $17.1 \pm 1.51$, respectively. The present study showed that salivary cortisol during acute exercise was significantly increased during exercise compared to that before exercise $(P<0.01)$ (Table 2$)$. The peak levels of salivary cortisol occurred at 14 min of exercise and showed decreased levels at $1 \mathrm{~h}$ post exercise $(P<0.01)$. The levels of salivary cortisol at $24 \mathrm{~h}$ after exercise were slightly higher than those measured during pre-exercise $(P<0.01)$. As can be seen from the data in Table 2 , the levels of salivary TNF- $\alpha$ increased steadily at 7 and 14 min of exercise compared to levels during preexercise $(P<0.01)$. However, the levels of TNF- $\alpha$ in saliva $1 \mathrm{~h}$ post exercise were decreased compared to levels at $14 \mathrm{~min}$ of exercise $(P<0.01)$. Furthermore, increased levels of nitrite in saliva could be detected at $14 \mathrm{~min}$ of exercise and remained the same until $24 \mathrm{~h}$ after exercise

Table 2 The levels of salivary cortisol, TNF- $\alpha$ and nitric oxide before, during and after acute exercise

\begin{tabular}{llll}
\hline Period of exercise & $\begin{array}{l}\text { Cortisol }(\mu \mathrm{g} / \mathrm{dl}) \\
\text { Mean }(\mathrm{SD})\end{array}$ & $\begin{array}{l}\text { TNF- } \alpha(\mathrm{pg} / \mathrm{ml}) \\
\text { Mean }(\mathrm{SD})\end{array}$ & $\begin{array}{l}\text { Nitrite }(\mu \mathrm{M}) \\
\text { Mean }(\mathrm{SD})\end{array}$ \\
\hline $\begin{array}{l}\text { Before } \\
\text { During }\end{array}$ & $0.15(0.04)$ & $4.93(0.55)$ & $2.03(0.04)$ \\
$\quad 7^{\text {th }}$ min & $0.45(0.06)^{\mathrm{a}}$ & $9.49(0.69)^{\mathrm{a}}$ & $1.65(0.04)^{\mathrm{a}}$ \\
$\quad 14^{\mathrm{th}}$ min & $0.70(0.04)^{\mathrm{a}, \mathrm{b}}$ & $14.16(0.93)^{\mathrm{a}, \mathrm{b}}$ & $3.01(0.06)^{\mathrm{a}, \mathrm{b}}$ \\
After & & & \\
$\quad 1 \mathrm{~h}$ & $0.35(0.03)^{\mathrm{a}, \mathrm{b}, \mathrm{c}}$ & $8.98(0.48)^{\mathrm{a}, \mathrm{b}, \mathrm{c}}$ & $3.43(0.06)^{\mathrm{a}, \mathrm{b}, \mathrm{c}}$ \\
$\quad 24 \mathrm{~h}$ & $0.20(0.02)^{\mathrm{a}, \mathrm{b}, \mathrm{c}, \mathrm{d}, \mathrm{d}}$ & $9.49(0.69)^{\mathrm{a}, \mathrm{b}, \mathrm{c}, \mathrm{d}}$ & $2.08(0.05)^{\mathrm{b}, \mathrm{c}, \mathrm{d}}$ \\
\hline
\end{tabular}

Significantly different at $P<0.01$ a before exercise, at 7 min of exercise,

${ }^{c}$ at 14 min of exercise, and ${ }^{\mathrm{d}} 1 \mathrm{~h}$ after exercise. $\mathrm{SD}=$ standard deviation 
$(P<0.01)$ (Table 2). Statistical analysis revealed that the levels of salivary NO at $24 \mathrm{~h}$ after exercise were not significant compared to those during pre-exercise $(P>$ $0.05)$.

\section{Discussion}

The results of the present study demonstrate that acute exercise elevates the levels of salivary cortisol, supporting the previous observations that exercise alters the levels of salivary cortisol $(2,3)$. Interestingly, the peak salivary cortisol elevation was seen at $14 \mathrm{~min}$ of exercise, indicating that the peak levels of cortisol production may actually occur at the point when subjects reach exhaustion during acute exercise. Therefore, the present study suggests that in order to obtain the precise profile of the alterations in cortisol levels due to acute exercise, the hormone levels both during and after acute exercise must be assessed.

Results of the present study show that elevated levels of TNF- $\alpha$ can also be detected in saliva during and after acute exercise. These results are similar to the alterations in serum levels of TNF- $\alpha$ after acute exercise as previously reported $(4,5)$. However, the precise mechanism by which the levels of salivary TNF- $\alpha$ were increased during acute exercise remains unclear. One of the possibilities is that elevated levels of other cytokines, such as M-CSF, during acute exercise may up-regulate the synthesis of TNF- $\alpha$ (12). This notion needs to be further clarified, since the present study did not assess the levels of salivary M-CSF.

Previous studies showed that acute exercise increases the levels of serum NO in humans $(7,8)$. The present study also found that salivary NO is augmented during acute exercise. Another study demonstrated, however, that salivary NO is increased by early exercise in athletes (9). The exact reason for the discrepancy between the present and previous reports (9) is unclear. Differences in the types of subjects and acute exercise protocol between the present and previous studies may be the cause. The source of elevated levels of salivary NO during acute exercise is unknown. It is possible that salivary NO may be derived from both muscle and endothelial cells as well as salivary glands during exercise $(6,13)$. If so, increased levels of salivary NO during exercise seen in the present study may also represent increased activities of NO synthase in both cell types and salivary glands. Furthermore, the exact mechanism by which acute exercise elevates levels of salivary NO, as seen in the present study, remains unclear. TNF- $\alpha$ is known to increase NO production by activating endothelial NO synthase (14). Thus, a possibility that increased salivary NO seen in the present study might be due to elevated activities of salivary TNF- $\alpha$ can not be ruled out.
The extrapolation of the present study in the physiological alteration following acute exercise remains speculative. Increased levels of salivary cortisol, TNF- $\alpha$ and NO during and after acute exercise may be associated with increased susceptibility to develop cardiovascular diseases as previously described $(4,6)$. Furthermore, since the altered profile of salivary composition due to exercise may represent that of systemic conditions (1), the results of the present study may support the view that altered host responses due to acute exercise may be observed from the saliva samples which are relatively easier and less stressful to obtain than serum samples.

In conclusion, the present study indicates that the levels of salivary cortisol, TNF- $\alpha$ and NO are increased during acute exercise in physically active males. Further studies, however, are needed to delineate whether or not salivary cytokines and NO may be used as biological markers to determine the host response to acute exercise.

\section{Acknowledgments}

The authors are grateful to Dr. Willy Pieter (School of Health Sciences, University Sains Malaysia) for statistical advice. This work was partially supported by an Intensified Research Priority Areas - Experimental Applied (IRPAEA) grant (Project No. 06-02-05-2074 EA 003) to W. Sosroseno, from The Malaysian Government.

\section{References}

1. Chicharro JL, Lucia A, Pérez M, Vaquero AF, Ureña R (1998) Saliva composition and exercise. Sports Med 26, 17-27.

2. O'Connor PJ, Corrigan DL (1987) Influence of short-term cycling on salivary cortisol levels. Med Sci Sports Exerc 19, 224-228.

3. Rudolph DL, McAuley E (1998) Cortisol and affective responses to exercise. J Sports Sci 16, 121-128.

4. Ostrowski K, Rohde T, Asp S, Scherling P, Pedersen BK (1999) Pro- and anti-inflammatory cytokine balance in strenuous exercise in humans. J Physiol 515 (Pt I), 287-291.

5. Nemet D, Oh Y, Kim HS, Hill M, Cooper DM (2002) Effect of intense exercise on inflammatory cytokines and growth mediators in adolescent boys. Pediatrics 110, 681-689.

6. Kingwell BA (2000) Nitric oxide-mediated metabolic regulation during exercise: effects of training in health and cardiovascular disease. FASEB J 14, 1685-1696.

7. Node K, Kitakaze M, Sato H, Koretsune Y, Katsube Y, Karita M, Kosaka H, Hori M (1997) Effect of 
acute dynamic exercise on circulating plasma nitric oxide levels and correlation to norepinephrine release in normal subjects. Am J Cardiol 79, 526-528.

8. Cuzzolin L, Lussignoli S, Crivellente F, Adami A, Schena F, Bellavite P, Brocco G, Benoni G (2000) Influence of an acute exercise on neutrophil and platelet adhesion, nitric oxide plasma metabolites in inactive and active subjects. Int J Sports Med 21, 289-293.

9. Panossian AG, Oganessian AS, Ambartsumian M, Gabrielian FS, Wagner H, Wikman G (1999) Effects of heavy physical exercise and adaptogens on nitric oxide content in human saliva. Phytomedicine 6, 1726.

10. Kasuya N, Kishi Y, Sakita S, Numano F, Isobe M (2002) Acute vigorous exercise primes enhanced NO release in human platelets. Atherosclerosis 161, 225-232.

11. Dunbar CC, Robertson RJ, Baun R, Blandin MF,
Metz K, Burdett R, Goss FL (1992) The validity of regulating exercise intensity by rating of perceived exertion. Med Sci Sports Exerc 24, 94-99.

12. Smith LL, Anwar A, Fragen M, Rananto C, Johnson R, Holbert D (2000) Cytokines and cell adhesion molecules associated with high-intensity eccentric exercise. Eur J Appl Physiol 82, 61-67.

13. Bodis S, Haregewoin A (1993) Evidences for the release and possible neural regulation of nitric oxide in human saliva. Biochem Biophys Res Comm 194, 347-350.

14. Barsacchi R, Perrota C, Bulotta S, Moncada S, Borgese N, Clamenti E (2003) Activation of endothelial nitric-oxide synthase by tumor necrosis factor-alpha: a novel pathway involving sequential activation of neutral sphingomyelinase, phosphatidylinositol-3' kinase, and Akt. Mol Pharmacol 63, 886-895. 